\title{
Produtividade e Eficiência Biológica de Sistemas Pecuários de Cria Diferindo na Idade das Novilhas ao Primeiro Parto e na Taxa de Natalidade do Rebanho no Rio Grande de Sul ${ }^{1}$
}

\author{
Virgínia Beretta ${ }^{2}$, José Fernando Piva Lobato ${ }^{3}$, Carlos Guilherme A. Mielitz Netto ${ }^{4}$
}

\begin{abstract}
RESUMO - Foi avaliada por intermédio da simulação a produtividade e eficiência de sistemas de criação de bovinos de corte no Rio Grande do Sul diferindo na idade das novilhas ao primeiro parto (IP, anos) e na taxa de natalidade (TN): "Sistema Tradicional" (ST) IP=4; "Sistema Melhorado" (SM) IP=3; e o "Sistema Um Ano" (SU) IP=2. Em cada sistema foi avaliado o efeito da TN variando entre 50 e $90 \%$. Foi usado um modelo estático determinístico. A resposta em produtividade e eficiência à redução na IP foi dependente da TN. No SM e SU foi determinada resposta curvilinear a aumentos na TN, atingindo uma produção máxima de 83,9 e 77,9 quilos de bezerro desmamado (BD)/ha com TN de 82,5 e 92,3\%, respectivamente. No ST a resposta foi linear, com aumentos de $6,1 \mathrm{~kg}$ BD/ha a cada $10 \%$ de aumento na TN. O custo energético por quilo BD produzido diminuiu linearmente, na medida em que a TN aumentou.
\end{abstract}

Palavras-chave: eficiência biológica, gado de corte, produtividade, simulação, sistemas

\section{Productivity and Biological Efficiency of Beef Cow-Calf Systems Differing in Age at First Calving and Herd Calving Rate in Rio Grande do Sul}

ABSTRACT - A simulation model was used to evaluate productivity and efficiency of beef cow-calf systems in Rio Grande do Sul State with different heifers first calving age (CA, years) and calving rates (CR): "Traditional System" (TS) CA=4; "Improved System (IS)" CA=3; "One Year System (OYS)" CA=2. In each system it was studied the effect of CR varying from 50 to $90 \%$. A statistic deterministic model was used. The effect of reducing CA on productivity and efficiency was dependent of CR. IS and OYS showed a quadratic response curve, with maximum production of 83,9 and $77,9 \mathrm{~kg}$ of weaned calf (WC)/ ha for CR of 82,5 and $92,3 \%$, respectively. TS response was linear, with increments of $6,1 \mathrm{~kg} \mathrm{WC} / \mathrm{ha}$ for each $10 \%$ increase in CR. Energetic cost per quilogram of WC was reduced linearly with increments in CR.

Key Words: biological efficiency, beef herd, productivity, simulation, systems

\section{Introdução}

O aumento da produtividade da pecuária de corte no Estado do Rio Grande do Sul tem sido colocado como uma alternativa de melhoria do resultado econômico das empresas dedicadas à atividade (PÖTTER et al., 1998; FRIES e ALBUQUERQUE, 1999). Esse processo passa pela melhoria dos indicadores de reprodução e produção do rebanho, congregando mudanças no nível tecnológico incorporado aos sistemas de produção, principalmente manejo alimentar e sanitário dos rebanhos, no melhoramento genético e a adequação de biotipos ao ambiente produtivo (LOBATO, 1995).

Pesquisas têm sido desenvolvidas no Rio Grande do Sul com o objetivo de identificar fatores determinantes do aumento da taxa de natalidade (GOTTSCHALL e LOBATO, 1996; SIMEONE e LOBATO, 1996; QUADROS e LOBATO, 1997) e da redução da idade das fêmas ao primeiro parto (BERETTA e LOBATO, 1996; ROCHA, 1997; PEREIRA NETTO e LOBATO, 1998). Porém, inexistem informações avaliando o impacto global no sistema de produção de mudanças conjuntas nestes dois indicadores.

Os diferentes fatores que compõem um sistema de produção interagem entre si, de forma que somente uma mudança parcial, afetando apenas uma categoria animal, poderá ter efeitos nas demais categorias do rebanho, dificultando a predição do impacto produtivo no conjunto do sistema. Assim, a avaliação do benefício de uma nova tecnologia deverá ser realizada considerando os seus efeitos em todo o sistema,

\footnotetext{
1 Parte da tese de Doutorado em Zootecnia apresentada à Universidade Federal do Rio Grande do Sul- UFRGS pelo primeiro autor.

2 Doutora, Departamento de Produção Animal e Pastagens, Faculdade de Agonomia, Universidade da República- Estação Experimental "M. A. Cassinoni" Cx.P 57 072, CP: 60 000, Paysandú - Uruguay. E.mail: asimeone@adinet.com.uy

3 PhD, Professor Adjunto IV, Dep. de Zootecnia, Fac. de Agronomia - UFRGS. Porto Alegre. Bolsista IA do CNPq. Caixa Postal, 776 - CEP 90.0001-970 - PortoAlegre. RS. E.mail: lobato@orion.ufrgs.br

4 Doutor, Professor Adjunto III, Dep. de Economia Rural, Fac. Economia - UFRGS - Porto Alegre, RS. E.mail: mielitz@vortex.ufrgs.br
} 
avançando além do impacto na categoria objeto da melhoria (BLACK et al., 1993). A modelagem e a simulação de sistemas têm sido propostos como instrumentos da pesquisa que permitem realizar este tipo de estudo, minimizando custos e reduzindo o tempo de avaliação (NAAZIE et al., 1999).

A quantificação produtiva da interação entre a idade ao primeiro parto e a taxa de natalidade pode priorizar a introdução de melhorias nos sistemas sobre uma base objetiva, fornecendo a informação necessária para a avaliação econômica, última variável de decisão em relação a uma inovação tecnológica.

O objetivo deste trabalho foi, através de um modelo quantitativo representativo de um sistema produtor de gado de corte no Rio Grande do Sul, avaliar o impacto na produtividade física e na eficiência biológica de mudanças nos indicadores reprodutivos (idade das novilhas ao primeiro parto e taxa de natalidade), e consequentemente, na intensidade de uso dos recursos do sistema.

\section{Material e Métodos}

Mediante simulação computacional, foi avaliada a produtividade e a eficiência biológica de três sistemas pecuários criadores diferindo na idade das fêmeas de reposicão ao primeiro parto (IP): "Sistema Tradicional" (ST), IP = 4 anos; "Sistema Melhorado" $(\mathrm{SM}), \mathrm{IP}=3$ anos, e "Sistema Um Ano" (SU), IP = 2 anos. Para cada um desses sistemas foram avaliadas mudanças na taxa de natalidade (TN) entre 50 e $90 \%$.

Foi utilizado um modelo estático determinístico representando o ciclo anual de cria dos diferentes sistemas em equilíbrio, montado com base num sistema de equações lineares simultâneas resolvidas por planilha eletrônica (BERETTA, 1999). Na Figura 1 é apresentado o diagrama correspondente à estrutura do modelo de programação. O modelo considerou as principais componentes biológicas de um sistema de produção de bovinos de corte: estrutura e tamanho de rebanho, disponibilidade e caracterísiticas dos recursos forrageiros, manejo alimentar e sanitário das diferentes categorias ao longo do ano. As componentes econômicas incluídas no modelo não foram consideradas neste trabalho.

O modelo foi dimensionado para uma área total de 1000 ha. A área da empresa pecuária foi utilizada como única restrição na estimação do estoque. Foi considerado ser o potencial produtivo dos recursos naturais o mesmo nos diferentes sistemas. Portanto, a produtividade não foi limitada pela dotação natural de recursos, mas pela adoção de tecnologia, o que viabilizou as comparações.

A estrutura e tamanho do rebanho é função da TN, da IP, da taxa de mortalidade, da taxa de utilização e reposição de touros, assim como do momento de venda das vacas de descarte. Para todos os casos foi considerado uma taxa de utilização de touros de $4 \%$, com $25 \%$ de reposicão anual, uma taxa de mortalidade de $1 \%$ para vacas, touros e novilhas, de $4 \%$ para bezerros no ST e SM, e de $5 \%$ no SU. Todas as vacas vazias em $1^{\circ}$ de abril saíram do rebanho de cria, sempre que a disponibilidade de novilhas de reposição foi igual ou maior a quantidade de vacas falhadas. Nessa avaliação foi fixada uma taxa de refugo mínima de $15 \%$.

A demanda energética para cada categoria animal do rebanho foi estimada com base nas equações do AGRICULTURAL AND FOOD RESEARCH COUNCIL - AFRC (1993) e o manejo alimentar definido mensalmente. A raça usada foi a Hereford, com um peso médio dos bezerros ao nascimento de 30 kg e à desmama aos 180 dias de $150 \mathrm{~kg}$, com uma produção média diária de leite para o período de lactação de 3,16 kg/vaca adulta (ROVIRA, 1996), sendo a produção das primíparas $80 \%$ desse valor. Com base nos dados reportados por ROVIRA (1996) e NUÑES DOMINGUEZ et al. (1991), no SU o peso à desmama de bezerros filhos de vacas primíparas aos dois anos foi considerado como sendo $12 \%$ inferior ao dos bezerros filhos de vacas primíparas aos três anos.

O peso vivo adulto das vacas é de $420 \mathrm{~kg}$, equivalente a uma condição corporal (CC) de três numa escala de cinco pontos $(1=$ magra; $5=$ gorda para abate). Para as vacas vazias foi considerado um peso médio de $339 \mathrm{~kg}$ no descarte. As variações em peso vivo assumiram equivalência de $50,8 \mathrm{~kg}$ por unidade de $\mathrm{CC}$, com base nos valores reportados por ORCASBERRO (1991). A CC nos distintos momentos fisiológicos variou dependendo da taxa de natalidade do rebanho. A CC ao parto e início de acasalamento foi definida a partir das relações encontradas por ORCASBERRO (1991) para $\mathrm{TN}<80 \%$ e porcentagem de prenhez $=-86,7+41,6 \mathrm{x}$ $\left(\mathrm{R}^{2}=0,996\right)$, e para $\mathrm{TN}>80 \%$ a porcentagem de prenhez $=60,66+4 x\left(\mathrm{R}^{2}=0,65\right)$.

As principais inovações tecnológicas consideradas, determinantes das mudanças nos coeficientes técnicos da fase de criação, foram: i) a introdução de 
1280 Rev. bras. zootec.

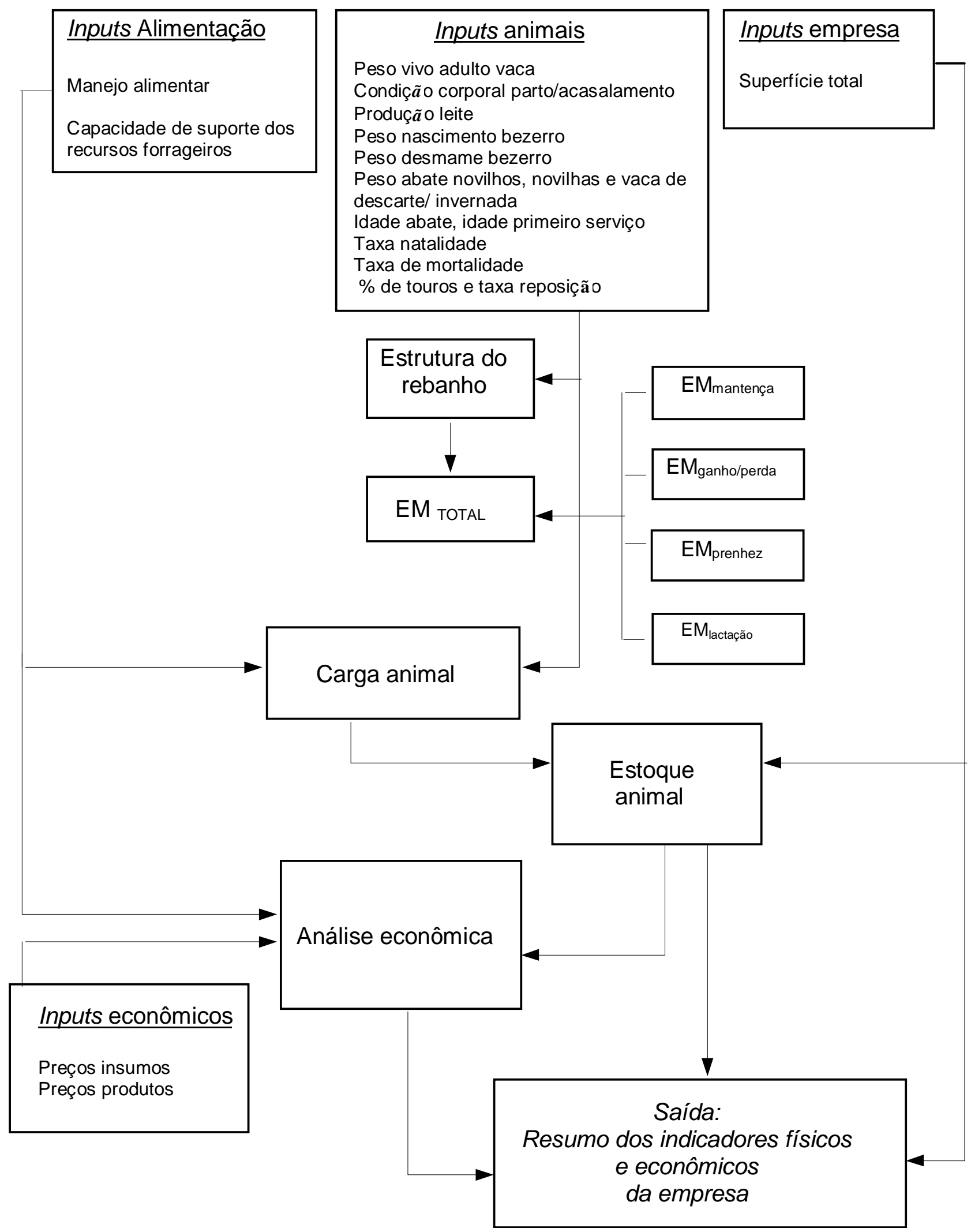

EM: energia metabolizável (metabolizable energy).

Figura 1 - Estrutura interna do modelo de programação para um sistema de ciclo completo de produção de carne bovina (Beretta, 1999).

Figure 1 - Model programm structure for a beef cattle production system (Beretta, 1999). 
pastagens melhoradas de clima temperado; ii) o ajuste da carga estacional em campo nativo; iii) o manejo da CC de vacas de cria ao longo do ciclo reprodutivo. A alimentação no ST foi considerada exclusivamente em campo nativo. A redução na IP de 48 para 36 ou 24 meses foi viabilizada a partir da utilização de pastagens cultivadas de ciclo hiberno/ primaveril, no período julho-novembro, até o primeiro acasalamento e durante o pré e o pós-parto das vacas primíparas (BERETTA e LOBATO, 1996; PEREIRA NETTO e LOBATO, 1998; PÖTTER et al., 1998). As vacas multíparas foram conduzidas sempre em campo nativo.

O campo nativo utilizado foi caracterizado por MARASCHIN (1998), com uma capacidade de suporte média para a estação de crescimento de setembro a maio, de $370 \mathrm{~kg}$ de peso vivo/ha com taxas máximas de ganho de até $0,517 \mathrm{~kg} / \mathrm{dia}$, e carga de 170 $\mathrm{kg} /$ ha durante o inverno para os animais manterem o peso. A utilização das pastagens melhoradas temperadas, consorciação de Lolium multiflorum (azevém), Trifolium repens (trevo branco) e Lotus corniculatus $c v$. São Gabriel (cornichão), foi de junho/julho a novembro, permanecendo posteriormente fechadas por cerca de 45/60 dias para favorecer a resemeadura e a perenidade das mesmas (LOBATO, 1985). Foi considerado uma vida produtiva média dessas pastagens de três anos. Baseado nas pesquisas de MÜLLER e PRIMO (1986), QUADROS e MARASCHIN (1987), ALBOSPINO e LOBATO (1993,1994), MORAES e MARASCHIN (1991), foi assumido uma capacidade de suporte para as pastagens melhoradas durante a estação de crescimento de 1,8 UA/ha (UA: unidade animal equivalente a $450 \mathrm{~kg}$ de peso vivo), com ganhos diários médios de $0,8 \mathrm{~kg} / \mathrm{animal}$.

A produtividadade física do sistema foi quantificada no modelo como os quilos de bezerro desmamado por hectare de pastejo, e como a soma dos quilos de bezerro desmamado mais os derivados da vaca de descarte, por unidade de área. A eficiência biológica, avaliada como a energia necessária por unidade de produto gerada no sistema, foi estimada como a relação entre a energia metabolizável (EM) consumida anualmente pelo rebanho de cria mais as fêmas de reposição e os quilos de peso vivo produzidos.

O modelo também contém informação descritiva para cada sistema. O uso da terra, a estrutura e o tamanho de rebanho, assim como a carga animal, avaliados no conjunto, permitem interpretar as possíveis diferenças entre os sistemas em termos de produtividade e eficiência.

\section{Resultados e Discussão}

A redução da IP determinou o aumento da superfície de pastagens no sistema de produção passando de $0 \%$ no $\mathrm{ST}$, baseado exclusivamente no campo nativo, para um máximo de $7,5 \%$ e $15,2 \%$ no SM e SU, repectivamente, quando a TN foi de $70 \%$. Quando essa foi de $80 \%$, a superfície de pastagens foi reduzida para $5,9 \%$ e $10,3 \%$, respectivamente, diminuindo as diferenças entre sistemas para 4,4 unidades percentuais. PÖTTER et al. (1998), reportaram que o fato de reduzir a idade ao primeiro parto de três para dois anos, na base de pastagens melhoradas, determina um aumento na superfície de pastagens necessárias da ordem de 6,05 unidades percentuais, quando a taxa de natalidade do rebanho foi de $80 \%$.

Consta da Tabela 1 a evolução da estrutura do rebanho conforme mudanças na $\mathrm{TN}$ em sistemas com diferentes IP das novilhas.

A IP afetou diretamente a proporção de novilhas de reposição em estoque, sendo o valor dependente da TN. Ocorreu uma relação de tipo quadrática entre as duas variáveis, atingindo a máxima proporção de novilhas quando a TN foi em torno de 64\% (ST: 63,6; SM: 64,3;SU: 64,7\%). Nesse ponto, no sistema com IP aos quatro anos, as novilhas representaram $53,1 \%$ do estoque, diminuindo para 44,4 e 28,7\% nos sistemas com IP aos três e dois anos, respectivamente. Quando a taxa de natalidade foi de $90 \%$, a proporção de novilhas reduziu-se para 35, 26 e $16 \%$ no ST, SM e SU, respectivamente.

A proporção de vacas de cria no estoque aumentou quanto menor foi a IP, ocorrendo um aumento linear na proporção de vacas prenhes na medida que aumentou a TN. Em síntese, situações extremas de baixa TN (50\%) e elevada IP (quatro anos), determinam uma estrutura de rebanho, onde apenas $33 \%$ do estoque corresponde a vacas de cria que concebem, $18 \%$ a vacas de cria que falham, mas que devem permanecer no rebanho por falta de novilhas de reposição, e $47 \%$ é constituído por novilhas de reposição de 0,5 a três anos de idade. Por outro lado, alta TN $(90 \%)$ e baixa IP (dois anos) resultaram em rebanhos com $81 \%$ de vacas parindo um bezerro todos os anos, em que não há vacas falhadas permanecendo no rebanho, e novilhas de reposição de 0,5 a um ano de idade constituem apenas $16 \%$ do estoque total.

O número de animais que foi necessário manter na empresa para cada 50 bezerros nascidos, por ano, diminuiu, à medida que os sistemas de produção se 
1282 Rev. bras. zootec.

Tabela 1 - Estrutura do rebanho em sistemas criadores conforme a idade ao primeiro parto das fêmeas: quatro (ST), três (SM) ou dois (SU) anos, e a taxa de natalidade do rebanho de cria adulto

Table 1 - Herd structure in breeding systems with different heifer's first calving age: four (TS), three (IS), or two years old (OYS), and calving rate of the mature herd

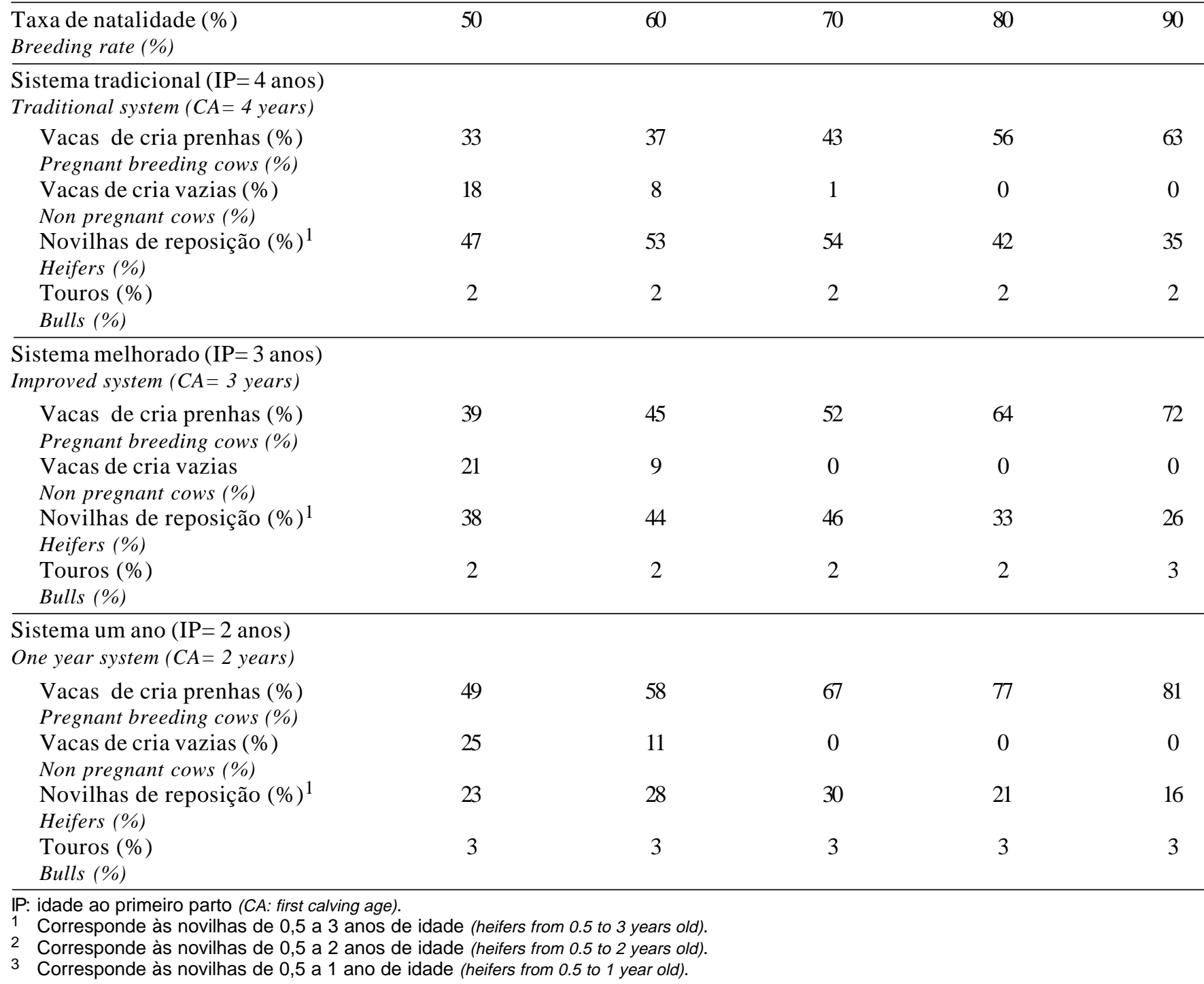

intensificaram, associado à diminuição na importância relativa das categorias de vacas falhadas e novilhas de reposição, o que aconteceu quando foi reduzida a IP e aumentada a TN (Figura 2). Enquanto no ST com $50 \%$ de TN foi necessário manter 180 animais para cada 50 bezerros nascidos, no SU com 90\% de TN foram necessários apenas 87 animais, uma redução de $51,7 \%$. A mesma tendência foi registrada para a àrea ocupada por cada um destes rebanhos: 202 ha no ST e 88 ha no SU para cada 50 bezerros nascidos, valor esse equivalente a $43,7 \%$ do registrado no ST (Figura 2).

Nos três sistemas avaliados, houve redução linear no tamanho do rebanho, conforme aumentou a TN. A área que acompanha esta evolução de rebanho apre- sentou a mesma tendência linear no ST, mas a relação foi curvilinear nos sistemas SM e SU, com decréscimos por unidade de aumento na TN. Estas diferenças têm a sua origem, basicamente, na introdução das pastagens melhoradas hiberno/primaveris nos sistemas SM e SU e no fato destas serem destinadas exclusivamente ao manejo alimentar das novilhas de reposição e das vacas primíparas.

A produtividade dos sistemas, avaliada em termos quilos de bezerro desmamado (BD)/ ha, aumentou conforme a IP diminuiu, porém, a magnitude da resposta foi dependente da TN do rebanho (Figura 3). Nos sistemas com primeiro parto aos dois e três anos, foi observada relação curvilinear entre TN e quilos de BD/ha, atingindo produção máxima de 83,9 


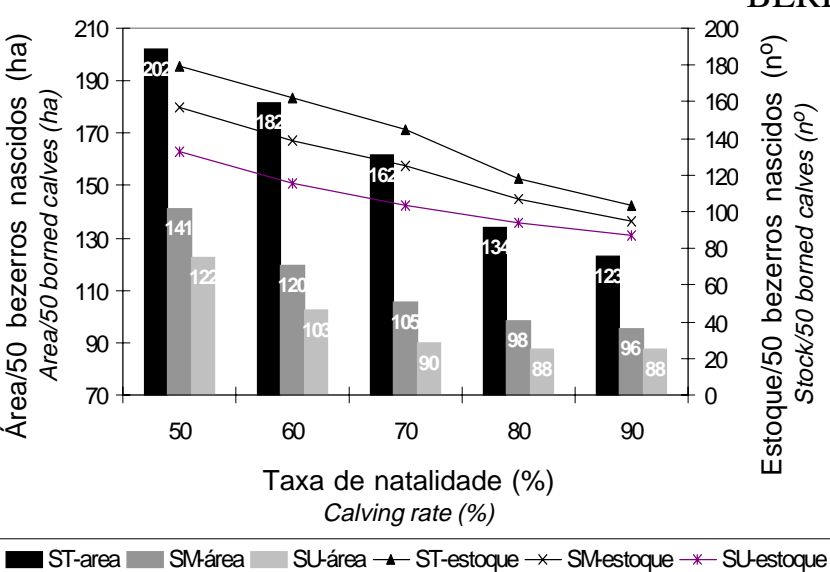

Figura 2 - Área e estoque animal/ 50 bezerros nascidos em sistemas criatório diferindo na idade ao primeiro parto das novilhas (quatro-ST, três-SM ou dois anos$\mathrm{SU})$ e na taxa de natalidade do rebanho de cria.

Figure 2 - Area and animal stock/ 50 calves born in cow-calf systems with different heifers first calving age (four-TS, three-IS or two years old-OYS) and herd calving rate.

e 77,9 $\mathrm{kg} \mathrm{BD} / \mathrm{ha}$, quando a $\mathrm{TN}$ foi de 82,5 e $92,3 \%$, no SU e SM, respectivamente. No ST, a relação foi linear, aumentando a produção de bezerros em $6,1 \mathrm{~kg} / \mathrm{ha}$ para cada $10 \%$ de aumento na $\mathrm{TN}$, atingindo um máximo de $59,6 \mathrm{~kg} / \mathrm{ha}$, quando a TN foi de $90 \%$.

No ponto de máxima produtividade para cada sistema, a redução da IP de quatro para três anos determinou aumento de 30,7\% nos $\mathrm{kg} \mathrm{BD} / \mathrm{ha}$, enquanto a redução da IP em mais um ano aumentou nos $\mathrm{kg} \mathrm{BD} / \mathrm{ha} \mathrm{em} \mathrm{7,7 \% .} \mathrm{A} \mathrm{máxima} \mathrm{resposta} \mathrm{à} \mathrm{mudan-}$ ça da IP encontrou-se em torno de $60-70 \%$ de natalidade, valores em que a porcentagem das categorias de novilhas no estoque se aproximou do máximo. Nesta faixa, a redução da IP de quatro para três, e de três para dois anos, traduziu-se em aumento dos quilos de BD/ha de 53,5 e 14,3\%, respectivamente.

A questão de o que priorizar na hora de decidir sobre as melhorias a incorporar ao sistema de produção dependerá da situação existente em cada caso. A interação existente entre TN e IP em sistemas com $50 \%$ de natalidade e novilhas parindo pela primeira vez aos quatro anos, mostrou que a redução da IP para três anos teve um impacto produtivo equivalente ao aumento da TN em $30 \%$. Porém, em sistemas com primeiro parto aos três anos, com rebanhos apresentando baixa $\mathrm{TN}$, foi possível aumentar a produtividade de forma significativa com base na melhoria da eficiência reprodutiva. Somente com altas taxas de

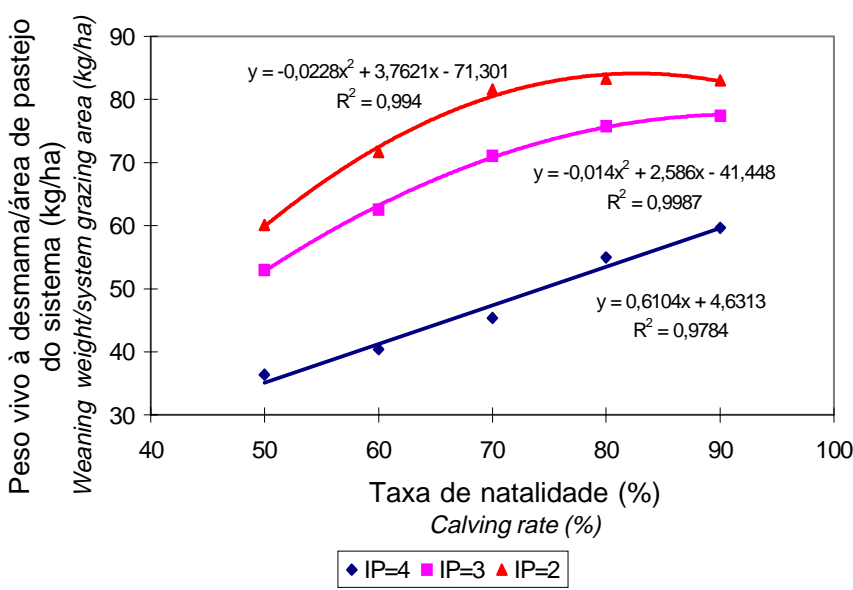

Figura 3 - Produção de peso vivo à desmama por hectare de pastejo em sistemas criatórios diferindo na idade ao primeiro parto (IP) das fêmeas (quatro-ST, três$\mathrm{SM}$ ou dois anos-SU) e na taxa de natalidade do rebanho de cria

Figure 3 - Calf weaning weight per grazing hectare in cow-calf systems differing in heifers first calving age (CA: fourTS, three-IS or two years old-OYS) and herd calving rate.

natalidade foi justificado, do ponto de vista biológico, adiantar em mais um ano a idade ao parto. Não há sentido em investir em um manejo intensivo da novilha e da vaca primípara, como o do sistema "Um Ano", quando ainda é possível aumentar a TN com base em medidas também técnicas, mas mais simples. Como exemplo básico temos o ajuste da carga animal conforme a disponibilidade média de matéria seca dos campos nativos e as exigências nutricionais de vacas adultas (TRUJILLO et al, 1993; SIMEONE e LOBATO, 1996; QUADROS e LOBATO,1997). A maior resposta foi observada quando a IP foi reduzida de quatro para três anos e a TN foi aumentada de 50 para $90 \%$, um aumento de $120,6 \%$ nos quilos de BD/ha. Porém, em sistemas já com IP aos dois anos, mudança da mesma magnitude nos coeficientes técnicos, representou um aumento de 56,3\% nos quilos de $\mathrm{BD} / \mathrm{ha}$.

O fato de considerar a vaca de descarte (VD) como um produto da cria vendido junto com bezerros desmamados modificou as relações entre TN e produtividade, descritas anteriormente. A TN que otimiza a produtividade e que é avaliada como quilos de BD mais os de VD (BD+VD)/ha de pastejo, foi inferior à que maximiza somente os $\mathrm{kg}$ de $\mathrm{BD}$, não diferindo muito entre sistemas com diferentes IP (ST: 72,8 vs 90\%; SM: 68,3 vs $92,3 \%$; SU: 67,8 vs $82,5 \%$ ). Taxas de natalidade em torno de $70 \%$ garantem a máxima 
1284 Rev. bras. zootec.

taxa de descarte de vacas e, assim, a máxima produção de peso vivo a partir desta categoria animal. $\mathrm{O}$ aumento da TN acima deste ponto determina que cada vaca de descarte seja substituída pela produção de um bezerro desmamado. No ponto de máxima produtividade em cada sistema, ainda é observada maior produção de peso vivo $(\mathrm{BD}+\mathrm{VD})$, quanto menor a idade ao primeiro parto $(\mathrm{ST}=87,5 ; \mathrm{SM}=135,9$; $\mathrm{SU}=160,3 \mathrm{~kg} / \mathrm{ha}$ ).

A energia consumida por quilo de BD diminuiu conforme aumentou a TN, sendo a magnitude da resposta dependente da IP das novilhas. Para cada $10 \%$ de aumento na TN, foi registrada redução linear na energia necessária por kg de BD de 8,81; 6,59 e 2,0 Mcal EM, no ST, SM e SU, respectivamente. No ST com $\mathrm{TN}=50 \%$ foram consumidas $84,3 \mathrm{Mcal} \mathrm{EM} / \mathrm{kg}$ BD. A redução da IP de quatro anos para três e, posteriormente, de três para dois anos reduziu a energia necessária por $\mathrm{kg} \mathrm{BD}$ produzido em 17,0 e $25,0 \%$, respectivamente. Essas mesmas mudanças, mas com $\mathrm{TN}=80 \%$, representaram melhorias de 13,2 e $8,4 \%$, respectivamente, no custo energético do quilo de peso vivo produzido à desmama. Para o SU com $80 \%$ de TN, as simulações indicaram que, para cada quilo de bezerro desmamado, eram consumidas no sistema 46,0 Mcal EM. Este valor encontra-se dentro da faixa de valores reportados em outros trabalhos avaliando sistemas de criação (GREEN et al, 1991; LAMB et al, 1992), representando redução de 45,4\% em relação ao custo energético de produção no ST com $50 \%$ de TN.

Diferenças na produção de peso vivo/ha na eficiência biológica entre sistemas são consequência das mudanças registradas na estrutura do rebanho e no uso da terra. A melhoria dos indicadores reprodutivos determinou que a área ocupada por categorias improdutivas, como as de vacas falhadas e de novilhas em crescimento, seja ocupada por categorias produtivas, basicamente por vacas e novilhas prenhes, aumentando a geração de produto por hectare de pastejo utilizada, e reduzindo o custo energético por quilo de peso vivo produzido. Isto ficou evidente no ST, em que foi registrada resposta linear positiva nos $\mathrm{kg} \mathrm{BD} / \mathrm{ha}$ com aumentos da TN.

Nos sistemas SM e SU, a incorporação das pastagens melhoradas de uso hiberno/primaveril aumentou a capacidade de suporte média da base forrageira do sistema, sendo o impacto global dependente do peso relativo que tenham no rebanho as categorias que as usam. Assim, o impacto produtivo da melhoria dos coeficientes técnicos nestes siste- mas foi resultante de mudanças conjuntas na estrutura do rebanho e no uso da terra.

$\mathrm{O}$ aumento da TN do rebanho de cria, foi associada a redução da carga animal em que são manejados os rebanhos de vacas adultas nos três sistemas $\left(\operatorname{Carga}(U A / h a)=0,621+0,052 T N-0,00006 T N^{2} ;\right)$, acompanhando as maiores exigências energéticas das vacas cuja CC deve aumentar afim de garantir a melhoria da TN (TRUJILLO et al., 1993; SIMEONE e LOBATO, 1996; QUADROS e LOBATO, 1997). No entanto, esta diminuição da carga animal foi compensada por maior produção por vaca. A substituição de vacas falhadas por vacas prenhas, produtoras de bezerros, aumentou a produtividade média do sistema. O fato de os sistemas SM e SU terem apresentado uma relação quadrática entre $\mathrm{TN}$ e quilos de $\mathrm{BD} / \mathrm{ha}$, mostrando grandes aumentos na faixa de TN entre 50 e $70 \%$, é explicado pelo aumento na capacidade de suporte média da base forrageira do sistema na medida que aumenta a necessidade de novilhas para reposição. Nesta situação, para cada vaca falhada que devia permanecer no sistema, na medida que aumenta a TN, a vaca foi substituída por uma novilha, a qual, além de ter menor peso vivo, foi manejada durante parte do ano em pastagem melhorada, liberando área de campo nativo que passa a ser ocupada por uma vaca prenhe. Esta taxa de substituição diminui em altas TN (acima de 70\%), na medida que a superfície de pastagens melhoradas começa a diminuir associada à redução das necessidades de novilhas de reposição.

Dada uma mesma TN, o maior impacto produtivo registrado ao reduzir a IP de quatro para três anos, em relação a diminuição de três para dois anos, foi também explicado pelas mudanças na estrutura do rebanho e no uso da terra. Ao passar do ST para o SM, tira-se a categoria de novilhas de dois a três anos, mas também as novilhas de 0,5 a dois anos passam a ocupar uma superfície proporcionalmente menor, já que, no segundo inverno pós-desmame, elas usarão pastagens melhoradas, liberando área de campo nativo a ser ocupada pelo rebanho de cria. Passar do SM a SU, embora signifique tirar as novilhas de um a dois anos do sistema, traz associado a necessidade de aumentar a área de pastagens melhoradas. As maiores taxas de ganho pós-desmama necessárias para atingir o peso de acasalamento aos 14-15 meses exigem uma menor carga no manejo desta categoria ( PÖTTER et al.,1998). Isto, somado a menor peso à desmama dos bezerros filhos das vacas primíparas aos dois anos, explica o 
menor impacto produtivo da redução da IP de três para dois anos em relação a mesma medida ao passar de quatro para três anos.

Estes resultados destacam a importância da avaliação da viabilidade de implantação de uma nova tecnologia considerando o funcionamento global do sistema de produção, e não apenas das categorias diretamente afetadas. Em termos gerais, pode ser dito que a resposta à incorporação de tecnologia ao processo produtivo é positiva, com incrementos decrescentes na medida que o sistema se torna mais intensivo. Este tipo de comportamento torna a empresa muito sensível as mudanças nos preços dos insumos, já que deve estar muito bem quantificada a resposta biológica de forma a poder avaliar a viabilidade econômica de qualquer empreendimento.

Diferenças entre TN que minimizam o custo energético por quilo de BD produzido e TN que maximizam a produção de peso vivo/ha, sujeitas às variações na importância relativa das VD na composição das vendas, colocam a questão de qual a TN ótima maximizadora do resultado econômico, valor que poderá mudar conforme as relações de preços de insumos e produtos.

\section{Conclusões}

Sistemas com primeiro parto das novilhas aos dois anos apresentaram maior produtividade em relação àqueles com o primeiro parto aos três anos, e estes a sistemas com primeiro parto aos quatro anos. Porém, a magnitude da resposta foi dependente da TN do rebanho de cria, sendo registrado o maior impacto para valores de TN entre 60-70\% e o mínimo para TN acima de $80 \%$. A uma mesma TN, a redução da IP de quatro para três anos representou aumento proporcionalmente maior do que reduzí-la de três para dois anos. O custo energético por unidade de produto foi menor, quanto menor a IP, e diminuiu de forma linear, na medida em que aumentou a TN.

O tamanho e a estrutura do rebanho, assim como o uso da terra, funcionaram como variáveis intermediárias, por meio das quais as mudanças nos coeficientes técnicos, e, portanto, a inovação tecnológica exerceram seus efeitos na produtividade e eficiência biológica do sistema. Maior TN e menor IP das novilhas reduziram a permanência de categorias improdutivas no sistema de produção, liberando áreas para outras categorias e reduzindo o custo energético por unidade de produto.

As relações quantitativas obtidas entre TN e IP marcaram um caminho tecnológico a percorrer. Partindo de sistemas com $50 \%$ de TN e IP das novilhas aos quatro anos, o primeiro grande impacto produtivo é a redução da IP para três anos, seguido da melhoria da eficiência reprodutiva do rebanho de cria. Somente quando se atingiu TN acima de $75 \%$, foi justificável reduzir em mais um ano a IP, como forma de elevar a produtividade do sistema.

A avaliação biológica dos efeitos de mudanças nos coeficientes técnicos dos sistemas criatórios permitirá avaliar do ponto de vista econômico a inovação tecnológica a ser usada, fator determinante a ser considerado na tomada de decisão em nível de empresa pecuária.

\section{Referências Bibliográficas}

AGRICULTURAL AND FOOD RESEARCH COUNCIL AFRC. 1993. Energy and protein requeriments of ruminants. Wallingford: Commonwealth Agricultural Bureaux International. $159 \mathrm{p}$.

ALBOSPINO, B.H.J.C., LOBATO, J.F.P. 1993. Efeitos do desmame precoce de bezerras no desempenho até os 24-26 meses de idade. R. Bras. Zootec., 22(6):1033-1043.

ALBOSPINO, B.H.J.C., LOBATO, J.F.P. 1994. Efeitos do desmame precoce de bezerros no desempenho até os 24-26 meses de idade. R. Bras. Zootec., 23(4):565-575.

BERETTA, V. Avaliação bioeconômica de sistemas alternativos de produção de gado de corte no Rio Grande do Sul. Porto Alegre, RS:UFRGS,1999. 204p. Tese (Doutorado em Zootecnia) - Programa de Pós-Graduação em Zootecnia, Universidade Federal do Rio Grande do Sul, 1999.

BERETTA, V., LOBATO, J.F. P.1996. Efeitos da ordem de utilização de pastagens melhoradas no ganho de peso e comportamento reprodutivo de novilhas de corte. R. Bras. Zootec., 25(6):1205-1215.

BLACK, J.L., DAVIES, G.T., FEMING, F.F. 1993. Rol of Computer Simulation in the Applications of Knowledge to Animal Industries. Aust. J. Agric. Res., 44(3):541-555.

FRIES, L.A., ALBUQUERQUE, L.G. Prenhez aos catorze meses: Presente e futuro. Elementos do compenente genético. In: REUNIÃO ANUAL DA SOCIEDADE BRASILEIRA DE ZOOTECNIA, 36, 1999, Porto Alegre. Anais... Porto Alegre, SBZ, 1999. p.419.

GOTTSCHALL, C., LOBATO, J.F.P. 1996. Comportamento reprodutivo de vacas de corte primíparas submetidas a três lotações em campo nativo. R. Bras. Zootec., 25(1):46-57.

GREEN, R.D., CUNDIFF, L.V., DICKERSON, G.E. et al. 1991. Output/Input differences among nonpregnant, lactating Bos indicus-Bos taurus and Bos taurus-Bos taurus F1 cross cows. J. Anim. Sci., 69(8):3156-3166.

LAMB, M. A., TESS, M.W., ROBINSON, O.W. 1992. Evaluation of mating systems involving five breeds for integrated beef production systems: I Cow-calf segment. J. Anim. Sci., 70(3):689-699.

LOBATO, J.F.P. 1985. Gado de cria. Tópicos. Porto Alegre: Adubos Trevo. 32p.

LOBATO, J.F.P. Produção e manejo de gado de corte. O estado da arte. In: REUNIÃO ANUAL DA SOCIEDADE BRASILEIRA DE ZOOTECNIA, 32, 1995, Brasília. Anais... 
1286 Rev. bras. zootec.

Brasília: SBZ, 1995. p.405-414.

MARASCHIN, G.E. Utilização , manejo e produtividade das pastagens nativas da região Sul do Brasil. In: Ciclo de palestras em produção e manejo de bovinos de corte. (3.:1998:Canoas). Resumos... III do Ciclo de Palestras em produção e manejo de bovinos de corte, Canoas, 4 a 7 de maio de 1998. Canoas. Ed. ULBRA, 1998. p.29-39.

MORAES, A., MARASCHIN, G. E. Produtividade animal de uma pastagem de azevém, trevo branco e capim pangola, submetida à diferentes pressões de pastejo. In: REUNIÃO ANUAL DA SOCIEDADE BRASILEIRA DE ZOOTECNIA, 28, 1991, João Pessoa. Anais... João Pessoa: SBZ, 1991. p.144.

MÜLLER, L., PRIMO, A.T.1986. Influência do regime alimentar no crescimento e terminação de bovinos e na qualidade da carcaça. Pesq. Agropec. Bras., 21(4):445-452.

NAAZIE, A., MAKARECHIAN, M., HUDSON, R.J. 1999. Evaluation of life-cycle herd efficiency in cow-calf systems of beef production. J. Anim. Sci., 77(1):1-11.

NUÑEZ-DOMINGUEZ, R., CUNDIFF, L.V., DICKERSON, G.E. et al. 1991. Lifetime production of beef heifers calving first at two vs. three years of age. J. Anim. Sci., 69(9):3467-3479.

ORCASBERRO, R. 1991. Estado corporal, control del amamantamiento y performance reproductiva de rodeos de cria. In: Pasturas y Producción Animal en Areas de Ganadería Extensiva. Montevideo, INIA.p.158-163. (Serie Técnica n.13)

PEREIRA NETO, O.A., LOBATO, J.F.P. 1998. Efeitos da ordem de utilização de pastagens nativas melhoradas no desenvolvimento e comportamento reprodutivo de novilhas de corte. R. Bras. Zootec., 27(1):60-65.

PÖTTER, L., LOBATO, J.F.P., MIELITZ NETTO, C.G.A. 1998. Produtividade de um modelo de produção para novilhas de corte primíparas aos dois, três e quatro anos de idade. R. Bras. Zootec., 27(3):613-619.
QUADROS, F.L.F., MARASCHIN, G.E. 1987. Desempenho animal em misturas de espécies forrageiras de estação fria. Pesq. Agropec. Bras., 22(5):535-541.

QUADROS, S.A.F., LOBATO, J.F.P. 1997. Efeitos da lotação animal no comportamento reprodutivo de vacas de corte primíparas. R. Bras. Zootec., 25(1):22-35.

ROCHA, M.G. Desenvolvimento e características de produção e reprodução de novilhas primíparas aos dois anos de idade. Porto Alegre, RS: UFRGS, 1997. 247p. Tese (Doutorado em Agronomia - Zootecnia) - Programa de Pós-Graduação em Agronomia, Universidade Federal do Rio Grande do Sul, 1997.

ROVIRA, J. 1996. Manejo reproductivo de los rodeos de cría en pastoreo. Montevideo: Hemisferio Sur. 288p.

SIMEONE, A., LOBATO, J.F.P. 1996. Efeito da lotação animal em campo nativo e do controle da amamantação no comportamento reprodutivo de vacas de corte primíparas. $R$. Bras. Zootec., 25(6):1216-1227.

TRUJILlO, A., BERETTA, V., FRANCO, J. et al. 1993. Presión de pastoreo en gestación avanzada y estado corporal de vacas Hereford al parto. Ciencia e Inv. Agr., 20(2):75.

Recebido em: 21/02/00

Aceito em: 15/05/01 